\title{
Aquaporin-4 upregulated expression in glioma tissue is a reaction to glioma-associated edema induced by vascular endothelial growth factor
}

\author{
LIJUAN YANG $^{1 *}$, XINGFU WANG $^{2 *}$, SHIMING ZHEN $^{3}$, SHENG ZHANG $^{2}$, DEZHI KANG ${ }^{3}$ and $_{\text {ZHIXIONG LIN }}^{3}$ \\ ${ }^{1}$ Department of Pharmacology, Fujian Medical University, Fuzhou, Fujian 350005; Departments of ${ }^{2}$ Pathology and \\ ${ }^{3}$ Neurosurgery, The First Affiliated Hospital, Fujian Medical University, Fuzhou, Fujian 350005, P.R. China
}

Received June 6, 2012; Accepted July 5, 2012

DOI: 10.3892/or.2012.1973

\begin{abstract}
Glioma-associated edema contributes significantly to morbidity and death in patients with glioma. It has been suggested that vascular endothelial growth factor (VEGF) and aquaporin-4 (AQP4) play important roles in gliomaassociated edema. However, the effect of VEGF on AQP4 expression is not clear. In this study, AQP4 expression was assayed in cultured glioma cells that express different amounts of VEGF, and AQP4 expression, vessel permeability and water content were assayed in glioma xenografts that express different amounts of VEGF. No difference in AQP4 expression was found between glioma cells expressing different VEGF amounts in vitro. However, AQP4 expression was increased in glioma tissue with increased VEGF, vessel permeability and water content. In conclusion, VEGF does not directly affect AQP-4 expression. The redistribution of AQP4 in glioblastoma cells is a reaction to vasogenic edema induced by VEGF for the purpose of facilitating reabsorption of excess fluid. The pattern of AQP4 expression in glioma provides new insights into the molecular changes occurring in glioma-associated edema and may help plan future therapeutic strategies.
\end{abstract}

Correspondence to: Dr Zhixiong Lin, Department of Neurosurgery, The First Affiliated Hospital, Fujian Medical University, Fuzhou, Fujian 350005, P.R. China

E-mail:1zx@mail.fjmu.edu.cn

*Contributed equally

Abbreviations: AQP4, aquaporin-4; VEGF, vascular endothelial growth factor; ZO, zonula occludens; JAM, junction associated molecules; ELISA, enzyme-linked immunosorbent assay; Q-PCR, quantitative polymerase chain reaction; HUVEC, human umbilical vein endothelial cells

Key words: glioma, edema, vascular endothelial growth factor, aquaporin-4

\section{Introduction}

Glioma progression can lead to glioma-associated brain edema, which is a significant source of morbidity and mortality (1). In-depth studies of molecular mechanisms of gliomaassociated edema have implicated vascular endothelial growth factor (VEGF), aquaporin-4 (AQP4), cyclooxygenase-2, zonula occludens (ZO), occludins, claudins, and junction associated molecules (JAM) in the process (2-6). VEGF (one of the most important factors promoting angiogenesis) is also responsible for plasma extravasation leading to peritumoral tissue edema, increased vessel permeability, and increase in the water content of glioma tissue $(7,8)$. Although some evidence is contradictory $(9,10)$, most accumulating evidence suggests the involvement of AQPs in the dynamics of brain edema formation or resolution (11). Mou et al found that the degree of peritumoral edema correlates with peritumoral AQP4 protein expression and that AQP4 expression correlates with VEGF and HIF-1 $\alpha$ expression (12). Another study showed that intracerebral VEGF injection dramatically upregulates AQP4 mRNA and protein in the perivascular space and glia limitans externa (13). Although there is a significant correlation between aquaporin-4 expression and the degree of cerebral edema, it is not clear whether increased aquaporin-4 expression enhances edema formation or clearance. The effects of VEGF on AQP4 expression may be important for understanding the molecular mechanism of edema. However, to our knowledge, there are no published reports on the effects of VEGF on AQP4 expression in glioma. The goal of the present study was to assess these effects and possibly provide a basis for developing novel therapeutic approaches for glioma-associated edema.

\section{Materials and methods}

Cell culture. Rat C6 glioma cells (Cell Biology Research Institute of Shanghai, Shanghai, China) and C6 cells with expression vectors containing antisense (C6/VEGF') VEGF164 cDNA or an empty vector $(\mathrm{C} 6 / \mathrm{vec})$ which were confirmed by assays for VEGF protein in cell culture supernatants and saved in our laboratory $(7,8)$ were cultured in RPMI-1640 medium (1640M) (Invitrogen, Carlsbad, CA, USA) supplemented with fetal calf serum (10\%). 
For cell proliferation assay, $2 \times 10^{4}$ cells were placed in a 6-well plate and were counted after 24, 48, 72, 96, 120 and $144 \mathrm{~h}$ culture by hemocytometer.

To measure VEGF secretion in vitro, $5 \times 10^{5}$ cells were placed in 6-well plates and treated with serum-free $1640 \mathrm{M}$. Medium was collected after $48 \mathrm{~h}$ of culturing. Debris was removed by centrifugation at $2000 \mathrm{x}$ g for $5 \mathrm{~min}$ and supernatant was collected for enzyme-linked immunosorbent assay (ELISA). A commercially available ELISA kit (R\&D Systems, Minneapolis, MN, USA) was used to detect mouse VEGF according to the manufacturer's recommendations. Each experiment was performed a minimum of three times.

VEGF activity assay. Confluence (80-90\%) of C6 cells, C6/vec cells and $\mathrm{C} 6 / \mathrm{VEGF}^{-}$cells were treated with serum-free $1640 \mathrm{M}$ for $48 \mathrm{~h}$. Media were collected and VEGF concentration were measured by ELISA. Human umbilical vein endothelial cells (HUVEC) $\left(2 \times 10^{4}\right.$, Cell Biology Research Institute of Shanghai) were placed in a 6-well plate and treated with $100 \mathrm{ng}$ of VEGF secreted from three C6 cell lines. The HUVEC cell growth curve was monitored by cell count.

Xenograft glioma animals. Male 4-6-week-old BALB/c (nu/nu) mice (SLAC, Shanghai, China) $(n=33)$ were randomized into three groups $(\mathrm{n}=11)$. Two individual clones of stable transfected C6 cells in the logarithmic growth phase were used for each construct (vector only, VEGF antisense), and the parental cell line was also studied. Two hundred microliters of cells (inserumfree medium with a final concentration of $7.5 \times 10^{6}$ cells $/ \mathrm{ml}$ ) were injected subcutaneously into the right inguinal area of the mice. Anesthetized mice were sacrificed with decapitation and tumors were removed from the athymic (nu/nu) mice at 20 days post-implantation. The animals were sacrificed and the tumors were removed and then quickly frozen in liquid nitrogen for further analysis. All procedures met the national guidelines for the care and use of laboratory animals and were approved by the Institutional Animal Care and Use Committee of the Fujian Medical University, Fujian, China.

Quantitative polymerase chain reaction ( $Q-P C R)$. Total RNA was isolated and cDNA was synthesized as has been described $(7,8)$. The sequences of primer sets were VEGF, forward: 5'-CCCAAGCTTATGAACTTTCTGCTCTCTTG-3', reverse: 5'-CGCGGATCCTCACCGCCTTGGCTTGTC-3'; AQP4, forward: 5'-GCATGAATCCAGCTCGATCCTTTGG-3' revese: 5'-AATGGGTGGCAGGAAATCTGAGGC-3'; $\beta$-actin, forward: 5'-GAGGCATCCTGACCCTGAAG-3', reverse: 5'-CATCACAATGCCAGTGGTACG-3'. The calculation of expression levels of VEGF and AQP4 was normalized by $\beta$-actin.

Immunohistochemistry. To detect VEGF and AQP4 expression in vitro, cells were fixed in $4 \%$ paraformaldehyde and blocked with $3 \%$ normal goat serum for $2 \mathrm{~h}$ at room temperature for immunocytochemistry analysis. To determine VEGF and AQP4 expression in vivo, anesthetized mice were decapitated, and tumor tissues were removed and quickly fixed in $10 \%$ formalin. For immunohistochemistry, $4-\mu$ m-thick sections were cut and rehydrated, treated with $0.3 \%$ hydrogen peroxide in methanol for $30 \mathrm{~min}$ to inactivate endogenous peroxidase, rinsed with $0.1 \mathrm{M}$ phosphate buffer (PB) for $10 \mathrm{~min}$, and exposed to blocking serum (3\% normal goat serum) for $2 \mathrm{~h}$ at room temperature.

Immunoreactions were performed as previously described (14). After incubation with anti-VEGF (1:150 dilution, United States Biological, Swampscott, MA, USA), and anti-AQP4 (1:150 dilution, Oncogene, Cambridge, MA, USA), the slices were rinsed with $0.1 \mathrm{M}$ PB and exposed to anti-rabbit IgG HRP (1:500, Maixin, Fuzhou, China). After an additional 10 -min rinse, the slices were treated with Vectastain ${ }^{\circledR}$ Elite $\mathrm{ABC}$ reagent (Maixin) for $30 \mathrm{~min}$ and developed with $\mathrm{DAB}$ detection kit (Maixin). The slices were counterstained by hematoxylin and mounted by Permount (Maixin).

Enzyme-linked immunosorbent assay (ELISA). To measure VEGF secretion in vitro, $5 \times 10^{5}$ cells were placed in 6-well plates and treated with serum-free $1640 \mathrm{M}$. Medium was collected after $48 \mathrm{~h}$ of culture. Debris was removed by centrifugation at 2,000 $\mathrm{x}$ g for $5 \mathrm{~min}$ and the supernatant collected for ELISA assay.

To measure VEGF levels in vivo, tumor tissues $(0.1 \mathrm{~g})$ were homogenized in Tris- $\mathrm{HCl}$ buffer $(25 \mathrm{mM}, \mathrm{pH}$ 7.6) containing $100 \mathrm{mM} \mathrm{NaCl}, 1 \mathrm{mM}$ EDTA, and $1 \mathrm{mM}$ phenylmethanesulfonyl fluoride (PMSF). Debris was removed by centrifugation at 2,000 $\mathrm{x}$ g for $5 \mathrm{~min}$, followed by centrifugation at $20,000 \mathrm{xg}$ for $20 \mathrm{~min}$, and supernatants were collected for ELISA assay. Protein concentrations were measured using Protein assay kit (Bio-Rad Laboratories, Hercules, CA, USA), and was normalized to a concentration of $1 \mathrm{mg} / \mathrm{ml}$. Series dilutions of samples with the highest and the lowest expected values were performed to determine VEGF expression level using commercial VEGF ELISA kit (R\&D Systems, Minneapolis, MN, USA) following the manufacturer's instructions. VEGF expression levels were calculated by a standard curve available from R\&D Systems. All experiments were performed in triplicate.

The water contents of tumor tissue assays. Referring to previously described studies $(7,8)$, the water contents of the tumor samples were measured and taken to represent the degree of edema. Tumor tissues from the same sample which was also sampled for assays of VEGF expression were immediately weighed on an electronic analytical balance to obtain the wet weight (WW). The samples were then dried in a gravity oven at $100^{\circ} \mathrm{C}$ for $24 \mathrm{~h}$ to obtain the dry weight (DW). Water content was expressed as a percentage of wet weight; the formula for calculation was (WW-DW)/WW x $100 \%$.

Tumor vessel permeability. Tumor-bearing mice received a $0.1-\mathrm{ml} / \mathrm{g}$ i.v. injection of Evans blue dye (1\% in saline; Sigma-Aldrich, St. Louis, MO, USA). After $6 \mathrm{~h}$ the animals were sacrificed and Evans blue was extracted from tumor as described (7). Briefly, tumors were removed and homogenized with $3 \mathrm{ml}$ of $N, N$-dimethylformamide (Sigma-Aldrich), and incubated at $57^{\circ} \mathrm{C}$ for $12 \mathrm{~h}$. The solutions were vortexed, then $2 \mathrm{ml}$ of $1 \mathrm{~N}$ hydrochloric acid $(\mathrm{HCl})$ were added, and the solutions were vortexed again, and then centrifuged at 2,500 rpm for $15 \mathrm{~min}$. Supernatant was collected and measured at $620 \mathrm{~nm}$ with a spectrophotometer (Beckman Coulter, Fullerton, CA, USA). Concentrations were calculated by using a standard curve for Evans blue dye. 


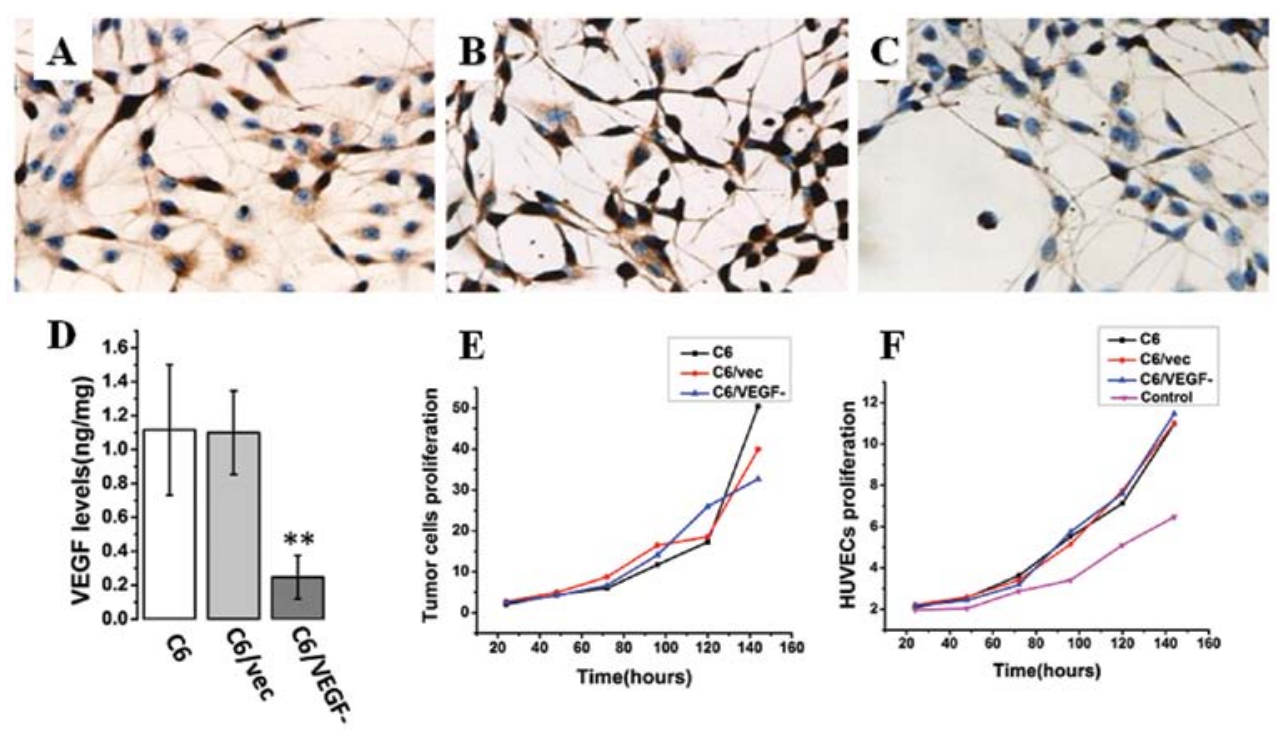

Figure 1. Expression of VEGF in glioma cells which express differential levels of VEGF in vitro. Immunocytochemical staining with antibody against VEGF antigen in C6 cells (A), C6/vec cells (B) and C6/VEGF- cells (C) after 48-h serum deprivation. (D) Enzyme linked immunosorbent assay (ELISA) of VEGF levels in condition media of $\mathrm{C} 6$ cells, C6/vec cells /and C6/VEGF- cells after 48-h serum deprivation. (E) Time course of cell proliferation of C6 cells (black), C6/vec cells (red) and C6/VEGF- cells (blue). (F) Time course of HUVEC cell growth curve with treatment of VEGF released from C6 cells (black), C6/vec cells (red), C6/VEGF- cells (blue) and non-treated (purple). ${ }^{*} \mathrm{P}<0.05 ; \mathrm{n}=4-6$.
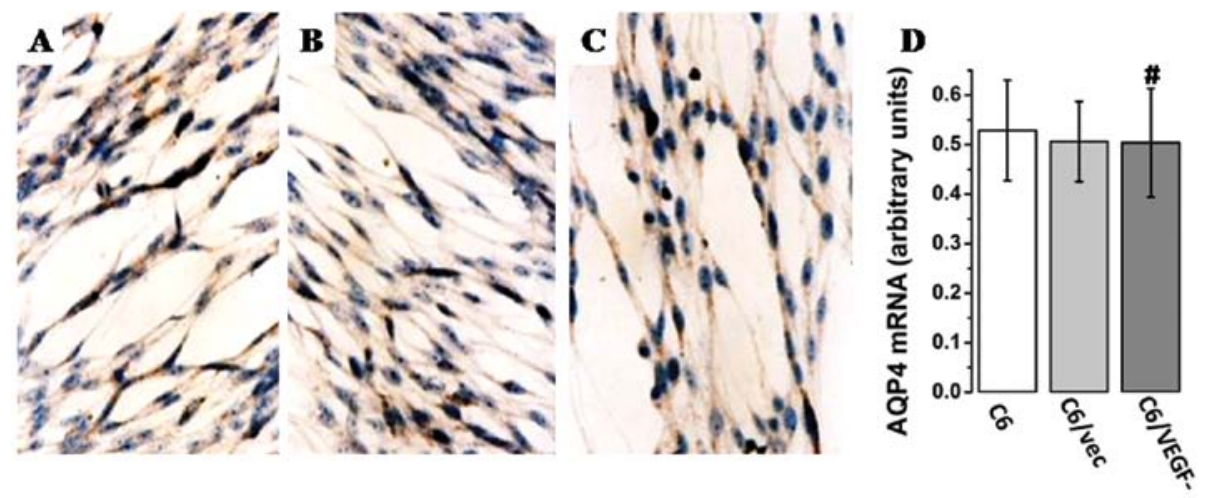

Figure 2. AQP4 expression in glioma cells which express differential levels of VEGF in vitro. Immunocytochemical staining with antibody against AQP4 antigen in C6 cells (A), C6/vec cells (B) and C6/VEGF- cells (C). The expression levels of AQP4 mRNA were assessed by quantitative PCR (D). ${ }^{~ P}>0.05$; $\mathrm{n}=4-6$.

Protein analysis. To analyze AQP4 expression in vivo, tumor tissues were homogenized in Tris- $\mathrm{HCl}$ buffer $(50 \mathrm{mM}, \mathrm{pH} 8.0)$ containing the protease inhibitor cocktail V (Calbiochem, San Diego, CA, USA). Homogenate ( $20 \mu \mathrm{g})$ proteins were separated by electrophoresis on 4-20\% SDS-PAGE gel and transferred onto Immobilon membranes (Millipore, Billerica, MA, USA). Western blot analyses were conducted using antibodies against AQP4 (1:100), and $\beta$-actin (1:2000, Neomarker, Fremont, CA, USA). Bands were visualized using an electrochemiluminescence (ECL) kit (Amersham Biosciences, Piscataway, NJ, USA).

Statistical analysis. SPSS 12.0 software (SPSS, Chicago, IL, USA) was applied for statistical data analysis. Data were analyzed by using one-way analysis of variance (ANOVA) followed by Dunnett's post hoc test for multiple comparisons to the control groups. Differences were considered significant at $\mathrm{P}<0.05$.

\section{Results}

Expression of VEGF in glioma cells in vitro. Immunostaining with VEGF antibody showed that endogenous VEGF level was lower in C6/VEGF- cells than in C6/vec and C6 cells after $48 \mathrm{~h}$ of serum deprivation (Fig. 1A-C). Similarly, the level of VEGF protein in the medium from $\mathrm{C} 6 / \mathrm{VEGF}^{-}$cells was significantly lower than that from C6/vec and C6 cells (Fig. 1D). To investigate the effect of antisense VEGF on tumor cell proliferation, the number of $\mathrm{C} 6 / \mathrm{VEGF}^{-}, \mathrm{C} 6 / \mathrm{vec}$, and $\mathrm{C} 6$ cells, respectively, placed into 6 -well plates $\left(2 \times 10^{4}\right.$ cells/well) were counted after 24, 48, 72, 96, 120 and $144 \mathrm{~h}$ in culture. Proliferation of C6/ $\mathrm{VEGF}^{-}$cells was found to be slower than that of the two control cells (C6/vec and C6; Fig. 1E), the morphology of all three cell lines was similar and remained unchanged (data not shown). However, the biological activity of VEGF released from different cell lines was not altered. Monitoring the growth of HUVEC cells treated with the same amount of VEGF secreted 
$\mathbf{A}$

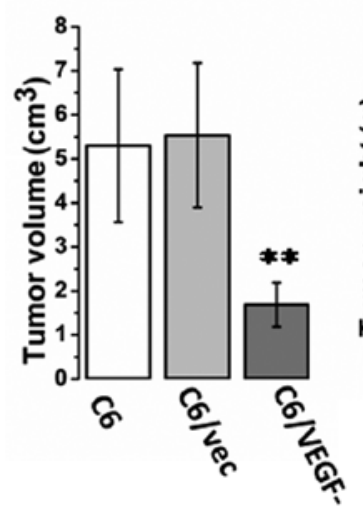

\section{B}

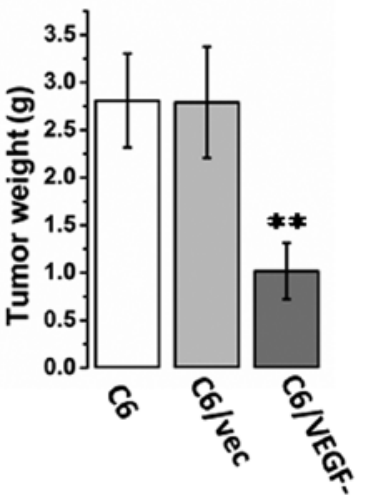

Figure 3. Characterization of xenograft glioma animals which express differential levels of VEGF. Analyses of tumor volume (A) and tumor weight (B) in $\mathrm{C} 6$ cell mice, $\mathrm{C} 6 / \mathrm{vec}$ cells and $\mathrm{C} 6 / \mathrm{VEGF}^{-}$cell mice. ${ }^{* *} \mathrm{P}<0.01 ; \mathrm{n}=7$.

from our three C6 cell lines revealed similar levels of HUVEC cell growth, regardless of the source of VEGF, and slower growth in the absence of VEGF (Fig. 1F).

Effect of glioma-derived VEGF on AQP4 expression in glioma cells in vitro. AQP4 expression in the three cell lines was assayed by RT-PCR and immunostaining. Similar AQP4 mRNA levels (Fig. 2A) were found in all three cell lines. In addition, the intensity of AQP4 immunoreactivity was similar in all lines after $48 \mathrm{~h}$ of serum deprivation (Fig. 2B-D). Thus, VEGF does not appear to have a direct role in AQP4 expression in glioma tumor cells.

Effect of VEGF on tumorigenesis. To evaluate the possible role of VEGF in tumorigenesis, mice were injected with glioma cells (C6, C6/vec, or C6/VEGF- cells) directly into the right inguinal area, and tumor size was measured. At 20 days after inoculation, tumor size (Fig. 3A) and tumor weight (Fig. 3B) were notably smaller in $\mathrm{C} / \mathrm{VEGF}^{-}$mice.

Expression of VEGF in tumors and the water content of tumor tissue. To confirm the expression of VEGF in vivo as well as in vitro, VEGF levels were determined in genetically modified
C6 cells by RT-PCR and ELISA. ELISA analysis (Fig. 4A) and RT-PCR (Fig. 4B) showed markedly lower level of VEGF mRNA and protein, respectively, in tumors from $\mathrm{C} 6 / \mathrm{VEGF}^{-}$ mice than tumors from $\mathrm{C} 6 / \mathrm{vec}$ and $\mathrm{C} 6$ mice. A common feature of malignant brain tumors is increased capillary permeability leading to edema. Assay of tumor water content showed that C6/VEGF tumors had a lower water content than either of the two control tumors (C6/vec and C6) (Fig. 4C). To confirm that the edema was attributable to vascular hyperpermeability, vascular extravasation was examined using a dye tracer. Vascular leakage was markedly reduced in the C6/ VEGF- tumors (Fig. 4D). Also, Pearson's correlation analysis found a correlation between water content and VEGF expression (Pearson's correlation, $\mathrm{r}=0.946 \mathrm{P}=0.00$ ).

Expression of AQP4 in tumors. Levels of AQP-4 appeared to be lower in C6/VEGF- tumors compared with $\mathrm{C} 6 \mathrm{G}, \mathrm{C6} / \mathrm{vecG}$ tumors by immunohistochemistry (Fig. 5A-C), and western blot analysis (Fig. 5D and E), and Pearson's correlation analysis show that AQP4 expression paralleled the level of VEGF expression $(\mathrm{r}=0.883, \mathrm{P}=0.00)$ and the water content $(\mathrm{r}=0.912$, $\mathrm{P}=0.00$ ) of glioma tissue. Thus, aquaporin- 4 expression in glioma tissue is suggested to be a reaction to glioma-associated edema induced by VEGF.

\section{Discussion}

Aquaporins (AQPs) are a family of water channel proteins that facilitate the flux of water through plasma membranes. AQP4, a mercury-insensitive water channel protein, is abundant in the central nervous system. It is localized in the blood-brain barrier around blood vessels and luminal membrane of ependymal cells, and its distribution in high density astrocytic foot processes is polarized. AQP4 is speculated to maintain the homeostasis of intracellular and extracellular water in the brain $(10,11,15)$. In addition, chemotherapy and radiotherapy for glioblastoma multiforme is reported to downregulate AQP4 expression, restoring its perivascular rearrangement and suggesting the potential role of AQP4 in the resolution of brain edema (16). Recent studies show that AQP4 is involved in cell migration and cytoskeleton organization $(15,17)$. Taken together, these findings suggest that AQP4 has a critical role in glioma malignancy.
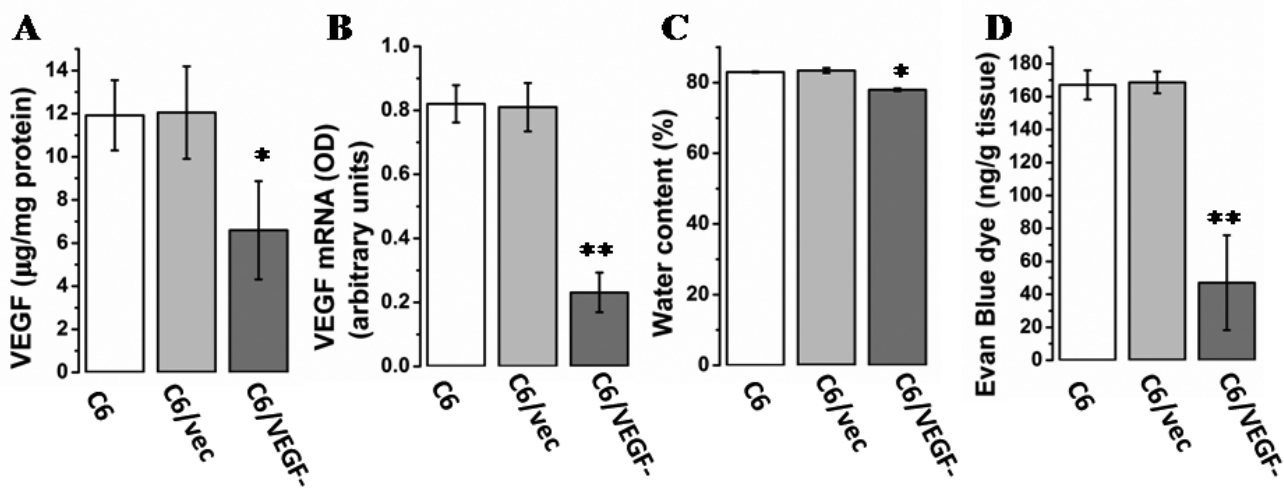

Figure 4. Expression of VEGF in tumors, vascular leakage and the water content of tumor tissue. VEGF protein and mRNA in tumor tissue were assessed by (A) ELISA and (B) Q-PCR. (C) Measurement of water content in tumor tissues. (D) Quantitative vascular leakage assessed by Evan blue dye tracer. " $\mathrm{P}<0.05$; ${ }^{* *} \mathrm{P}<0.01 ; \mathrm{n}=4-6$. 

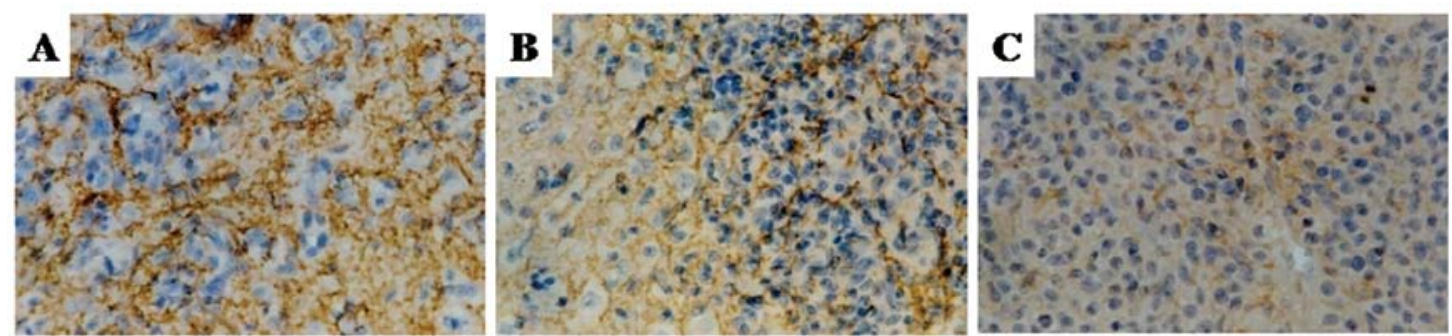

D

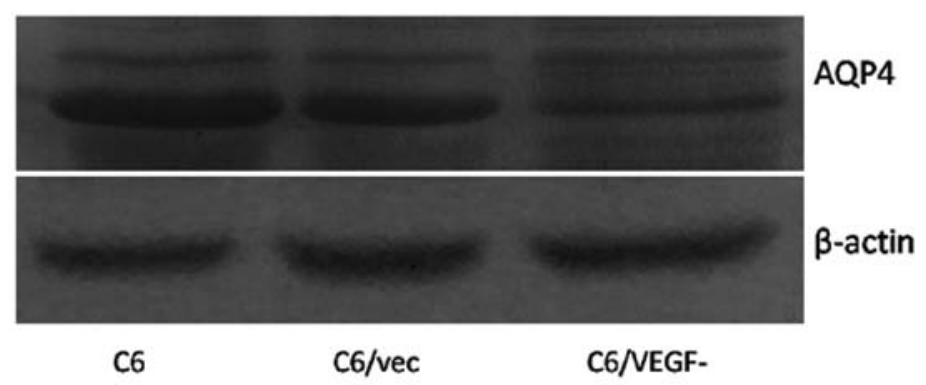

$\mathbf{E}$

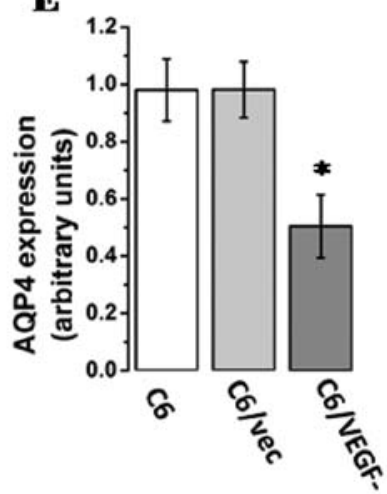

Figure 5. AQP4 analysis in xenograft glioma tissue which express differential levels of VEGF. Immunocytochemical staining with antibody against AQP4 antigen in C6 cell glioma (A), C6/vec cell glioma (B) and C6/VEGF- cell glioma (C); AQP4 western blot analysis (D); quantitative expression levels of AQP4 (E). ${ }^{*} \mathrm{P}<0.05 ; \mathrm{n}=4$.

Breakdown of the blood-brain barrier (BBB) has been linked to upregulation of AQP4 expression. The increased AQP4 expression in high grade astrocytomas may facilitate the flow of edema fluid (18). The pattern of AQP4 expression in human gliomas, AQP4 overexpression in glioma cells, and AQP4 localization on astrocytic end-feet are associated with disturbance of the blood-brain barrier (19). The redistribution of AQP4 in glioblastoma cells is believed to facilitate reabsorption of excess fluid and to be a reaction to vasogenic edema stemming from the breakdown of the BBB (20).

Mou et al hypothesized that AQP4 is positively regulated by VEGF (12). Rite et al (13) found that intracerebral injection of VEGF induces an increase in AQP4, but in our study, VEGF did not directly affect AQP-4 expression. The most important factor regulating the function and expression of AQP4 is osmotic pressure. Studies have shown that VEGF may alter vascular permeability and affect osmotic pressure changes (21). VEGF can increase neovascular permeability and promote the extravasation of plasma protein and fibrinogen into intracellular spaces. In glioma, VEGF is one of the most important factors promoting angiogenesis in the tumor. With growth of the tumor, increase in vascular permeability due to neovascularization causes extensive damage to the BBB integrity, and a large number of macromolecules in plasma enter the interstitial space, where they produce an obvious change in osmotic pressure. Therefore, VEGF is an important factor affecting osmotic pressure within glioma tissue. Therefore, although AQP4 was associated with brain edema formation, we presume that upregulated expression and redistribution of AQP4 in glioblastoma cells is a reaction to VEGF-induced vasogenic edema and a response that ameliorates or prevents cytotoxic brain edema by facilitating reabsorption of excess fluid.
In summary, VEGF does not directly affect AQP-4 expression. The redistribution of AQP4 in glioblastoma cells is a reaction to VEGF-induced vasogenic edema and facilitates reabsorption of excess fluid. AQP4 induction might be a promising approach in vasogenic brain edema prevention and treatment. Further studies are needed to understand its functional role.

\section{Acknowledgements}

We thank Professor Lin Xu (Research Center of Molecular Medicine, Fujian Medical University) for help in primer design and Professor Tian Jun and Professor Hu Zhi-jian (Public Health School, Fujian Medical University) for assistance in data processing and statistical analysis. This study was supported by a grant from National Natural Science Foundation of China (no. 30973083).

\section{References}

1. Papadopoulos MC, Saadoun S, Davies DC and Bell BA: Emerging molecular mechanisms of brain tumour oedema. Br J Neurosurg 15: 101-108, 2001.

2. Dobrogowska DH,Lossinsky AS, Tarnawski M and Vorbrodt AW: Increased blood-brain barrier permeability and endothelial abnormalities induced by vascular endothelial growth factor. J Neurocytol 27: 163-173, 1998.

3. Wang W, Dentler WL and Borchardt RT: VEGF increases BMEC monolayer permeability by affecting occludin expression and tight junction assembly. Am J Physiol Heart Circ Physiol 280: H434-H440, 2001.

4. Yool AJ, Brown EA and Flynn GA: Roles for novel pharmacological blockers of aquaporins in the treatment of brain oedema and cancer. Clin Exp Pharmacol Physiol 37: 403-409, 2010.

5. Badie B, Schartner JM, Hagar AR, et al: Microglia cyclooxygenase-2 activity in experimental gliomas: possible role in cerebral edema formation. Clin Cancer Res 9: 872-877, 2003. 
6. Vorbrodt AW and Dobrogowska DH: Molecular anatomy of intercellular junctions in brain endothelial and epithelial barriers: electron microscopist's view. Brain Res Brain Res Rev 42: 221-242, 2003.

7. Lin ZX, Yang LJ, Huang Q, et al: Inhibition of tumor-induced edema by antisense VEGF is mediated by suppressive vesiculovacuolar organelles (VVO) formation. Cancer Sci 99: 2540-2546, 2008.

8. Yang LJ, Lin ZX, Huang Q, et al: Effect of vascular endothelial growth factor on remodeling of C6 glioma tissue in vivo. J Neurooncol 103: 33-41, 2011.

9. Manley GT, Fujimura M, Ma T, et al: Aquaporin-4 deletion in mice reduces brain edema after acute water intoxication and ischemic stroke. Nat Med 6: 159-163, 2000.

10. Papadopoulos MC, Manley GT, Kfishna S and Verkman AS: Aquaporin-4 facilitates reabsorption of excess fluid in vasogenic brain edema. FASEB J 18: 1291-1293, 2004.

11. Zador Z, Bloch O, Yao X and Manley GT: Aquaporins: role in celebral edema and brain water balance. Prog Brain Res 161: 185-194, 2007.

12. Mou K, Chen M, Mao Q, Wang P, Ni R, Xia X and Liu Y: AQP-4 in peritumoral edematous tissue is correlated with the degree of glioma and with expression of VEGF and HIF-alpha. J Neurooncol 100: 375-383, 2010.

13. Rite I, Machado A, Cano J and Venero JL: Intracerebral VEGF injection highly upregulates AQP4 mRNA and protein in the perivascular space and glia limitans externa. Neurochem Int 52: 897-903, 2008
14. Lin ZX, Yang LJ, Huang Q and Fu J: Activated vascular endothelia regulate invasion of glioma cells through expression of fibronectin. Chin Med J 123: 1754-1761, 2010.

15. Ding T, Gu F, Fu L and Ma YJ: Aquaporin-4 in glioma invasion and an analysis of molecular mechanisms. J Clin Neurosci 17: 1359-1361, 2010.

16. Nico B, Mangieri D, Tamma R, et al: Aquaporin-4 contributes to the resolution of peritumoural brain oedema in human glioblastoma multiforme after combined chemotherapy and radiotherapy. Eur J Cancer 45: 3315-3325, 2009.

17. McCoy E and Sontheimer H: Expression and function of water channels (aquaporins) in migrating malignant astrocytes. Glia 55: 1034-1043, 2007

18. Saadoun S, Papadopoulos MC, Davies DC, Krishna S and Bell BA: Aquaporin-4 expression is increased in oedematous human brain tumours. J Neurol Neurosurg Psychiatry 72 : 262-265, 2002.

19. Davies DC: Blood-brain barrier breakdown in septic encephalopathy and brain tumours. J Anat 200: 639-646, 2002.

20. Warth A, Mittelbronn M and Wolburg H: Redistribution of the water channel protein aquaporin- 4 and the $\mathrm{K}^{+}$channel protein Kir4.1 differs in low- and high-grade human brain tumors. Acta Neuropathol 109: 418-426, 2005.

21. Fu BM and Shen S: Structural mechanisms of acute VEGF effect on microvessel permeability. Am J Physiol Heart Circ Physiol 284: H2124-H2135, 2003. 